\title{
Effect of microneedle radiofrequency on scars of transcutaneous lower blepharoplasty
}

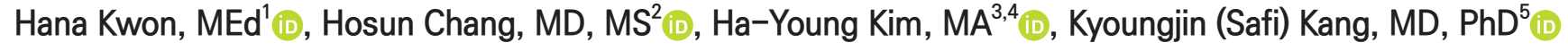 \\ ${ }^{1}$ Korean College of Cosmetic Surgery, Seoul, Rep. of Korea \\ ${ }^{2}$ Medicastle Clinic, Cheonan, Rep. of Korea \\ ${ }^{3}$ Interdisciplinary Program in Bioengineering, Seoul National University, Seoul, Rep. of Korea \\ ${ }^{4}$ Department of Plastic and Reconstructive Surgery, Seoul National University Bundang Hospital, Seongnam, Rep. of Korea \\ ${ }^{5}$ Department of Plastic Surgery, Seoul Face 21 Dental Hospital, Seoul, Rep. of Korea
}

Despite the popularity of transcutaneous lower blepharoplasty (TLB) for the correction of the aged lower lids, ectropion, scar long the incision line, persistent skin laxity with fine wrinkles remain to be a huge setback associated with the procedure. With the advance of recent technologies, scar treatment methods using various kinds of devices have been widely adopted. Among them, fractional microneedle radiofrequency (FMR) has been proven to be highly effective on the improvement of acne scars, widened pores, and skin laxity by means of soft tissue regeneration. A fractional microneedle device called the Inus ${ }^{\mathrm{TM}}$ developed in Korea exhibits unique properties of delivering bipolar radiofrequency current through specialized vacuum edge line for optimal needle insertion into highly sensitive areas such as the eyelid and the neck. Usage of the Inus ${ }^{\top \mathrm{M}}$ to treat patients experiencing complications from TLB have shown remarkable improvement in various aspects not only limited to the improved appearance of ectropion, scars, wrinkles, skin thickness and laxity, but also the contour of the eyelid and the overall facial volume, and shape. The report will delineate findings from three clinical cases that suggest the efficacy and safety of FMR on the rejuvenation of the lower lids.

Keywords: fractional microneedle radiofrequency; lower lid; rejuvenation; scar; transcutaneous lower blepharoplasty

\section{Introduction}

Transcutaneous lower blepharoplasty (TLB) is an invasive surgery that is most commonly used for the treatment of the aged lower eyelid [1]. It is commonly performed in patients at middle to late adulthood, despite the expected complications of linear or depressed scar formations along the incision lines of TLB. Although a majority of the scars resolve on their own within 6 months of surgery, some may persist even after the application of an anti-scar ointment. Patients tend to resort to scar revision surgery as the last option. Increased patient dissatisfac- tion has been observed in many patients after TLB.

Non-ablative fractional microneedle radiofrequency (FMR) devices are known to be effective for the treatment of periorbital malar bags and wrinkles, inflammatory acne vulgaris and related scars, facial large pores, primary axillary hyperhidrosis, striae, as well as skin laxity [2,3]. However, there have been no reports on the rejuvenation effect of bipolar FMR devices on the eyelids, except for one clinical case in which monopolar microneedle radiofrequency $(\mathrm{RF})$ device was employed to treat lower eyelid fat bulging [4]. In contrary to existing devices, the Inus ${ }^{\mathrm{TM}}$ Unit was developed by Medromed Ltd. (Seoul, Korea)

Received November 17, 2021; Revised December 13, 2021; Accepted December 13, 2021

Corresponding author: Kyoungjin (Safi) Kang

E-mail: safikccs@pascal-world.com

This is an Open Access article distributed under the terms of the Creative Commons Attribution Non-Commercial License (http://creativecommons.org/licenses/by-nc/4.0), which permits unrestricted non-commercial use, distribution, and reproduction in any medium, provided the original work is properly cited.

Copyright $@ 2021$ Korean Society of Korean Cosmetic Surgery and Medicine (KSKCS \& KCCS). 
for the purpose of rejuvenating the eyelids. It is equipped with a specialized apparatus known as the vacuum edge line that allows easy and precise insertion of microneedles into laxed skin with a reduced degree of pain and swelling during the procedure (Fig. 1).

To date, no studies have been conducted on the effect of needle RF on scar improvement after the TLB procedure. This case report demonstrates the rejuvenation effect of the Inus ${ }^{\mathrm{TM}}$ Unit on depressed scars in three patients post-TLB. Microneedles of depth $1.0 \mathrm{~mm}$ or $0.8 \mathrm{~mm}$ were used to apply RF of 0.5 or 2.0 $\mathrm{MHz}$ respectively, to the entire lower lid during three sessions, held at intervals of 2 weeks each. Correction of depressed scars, reduced appearance of wrinkles, and increased thickness of the lower lid skin were noticeable to the eye. Moreover, due to the improvement of lid laxity and ectropion, a lifting effect was observed on the lower lid. These cases suggest the high potential of FMR as a therapeutic method for the correction of TLBinduced depressed scars and for the overall enhancement of

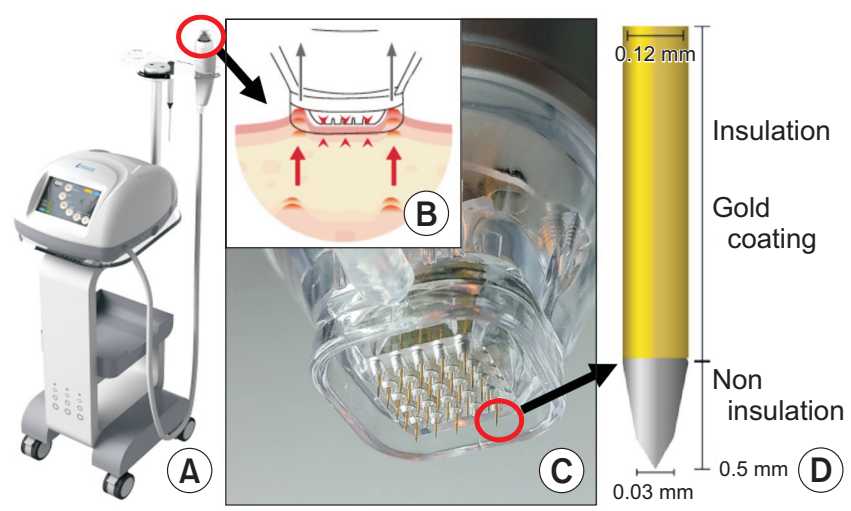

Fig. 1. Components of a non-ablative bipolar fractional microneedle radiofrequency device (Inus ${ }^{\mathrm{TM}}$ ). A main body and a hand piece (A), schematic diagram of action using a temporary tip with peripheral vacuum edge line (B), and image of the twenty-five numbers of fractional needles located in the center of the tip (C). (D) The entire length of the needle is $3.5 \mathrm{~mm}$ and its thickness is $0.12 \mathrm{~mm}$. The distal part, $0.5 \mathrm{~mm}$ in length, is non-insulated, whereas the proximal part, $3.0 \mathrm{~mm}$ in length, is insulated with gold. skin rejuvenation of the lower lid.

Inus ${ }^{\mathrm{TM}}$ was applied to three patients with depressed scars after TLB. The parameters used are listed in Table 1.

Topical anesthesia was applied to the treated area for at least 30 minutes. Following disinfection using benzalkonium chloride solution (10\%), a temporary Inus ${ }^{\mathrm{TM}}$ tip with 25 microneedles was gently placed in contact with the skin of the orbital and the preorbital regions, including the proximal skin of the midface, temporal hollows, and brows in a vertical orientation. As the peripheral skin was drawn into the vacuum edge line by negative pressure, 25 microneedles were simultaneously inserted into the central skin (Fig. 1; Supplementary Video). Throughout the procedure, $3.0 \mathrm{ml}$ of multi-peptide mesotherapy solution (MISTCELL; Meso Pharmaco., Ltd., Seoul, Korea) was continuously applied to the lower lid skin. After the procedure, the patient was advised to apply antibiotic ointment to the treated area for 2 days. Pre- and postoperative photographs were taken during the first visit of the patients and again, 2 weeks after their third session, resulting in a total of 8 weeks of
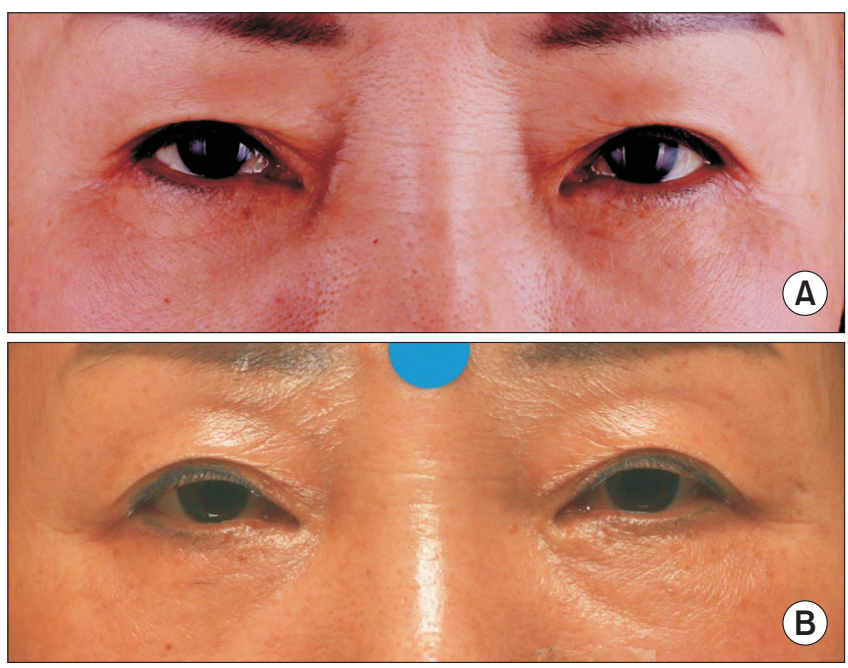

Fig. 2. Photos of case 1. (A) Before and (B) 2 weeks after the third sessions of Inus ${ }^{\mathrm{TM}}$ (8 weeks after the first session).

Table 1. Inus ${ }^{\mathrm{TM}}$ parameters of the fractional radiofrequency microneedle in the authors' study

\begin{tabular}{|c|c|c|}
\hline \multirow{2}{*}{ Parameter } & $0.5 \mathrm{MHz}$ bipolar mode & 2.0 MHz bipolar mode \\
\hline & First pass & Second pass \\
\hline Treatment site & \multicolumn{2}{|c|}{ Entire lower lid, lid cheek junction, and lateral canthal area } \\
\hline Depth of needle (mm) & 1.0 & 0.8 \\
\hline Intensity of power (W) & 0.88 & 0.66 \\
\hline Pulse width (ms) & 130 & 140 \\
\hline Suction interval/level & $0.5 \mathrm{~s} / 1$ & $0.5 \mathrm{~s} / 1$ \\
\hline Mean tip count & \multicolumn{2}{|c|}{ Range, 90-110/each eyelid } \\
\hline
\end{tabular}


follow-up. In addition, each patient was given an opportunity to review the manuscript and consented to its publication.

\section{Case report}

A 59-year-old female who underwent TBL was treated with Inus ${ }^{\mathrm{TM}}$ for correction of scars in the lower eyelids. Pretarsal atrophy with depressed scars was seen along the previous incision, and fine wrinkles were well-developed (Fig. 2A). Following treatment, there was a reduction in the thickening of the lower lid skin and in the appearance of wrinkles. Volume restoration was achieved in most areas except for the central pretarsal regions (Fig. 2B). Insufficient contact of the tip in the central pretarsal regions may result from the use of a non-needle vacuum edge line or inappropriate depths of the needles.

A 61-year-old female who presented with an unnatural appearance of pretarsal atrophy with scars along the previous TBL-induced incision lines was treated with Inus ${ }^{\mathrm{TM}}$ (Fig. 3). Laxed, deflated and wrinkled lower lids with ectropion were observed (Fig. 3A).

Post-treatment, there was a significant improvement in the appearance of linear and depressed scars and wrinkles were significantly improved. The lower lids appeared to be significantly volumized and lifted in the superolateral direction, and its effect was comparable to that of the revision blepharoplasty supplemented with midface lifting and fat facial grafting. Surprisingly, the contour of the lower lids was modified from a round to a straighter shape, the lid position shifted upward, the lateral canthal angles decreased, and ectropion and wrinkles
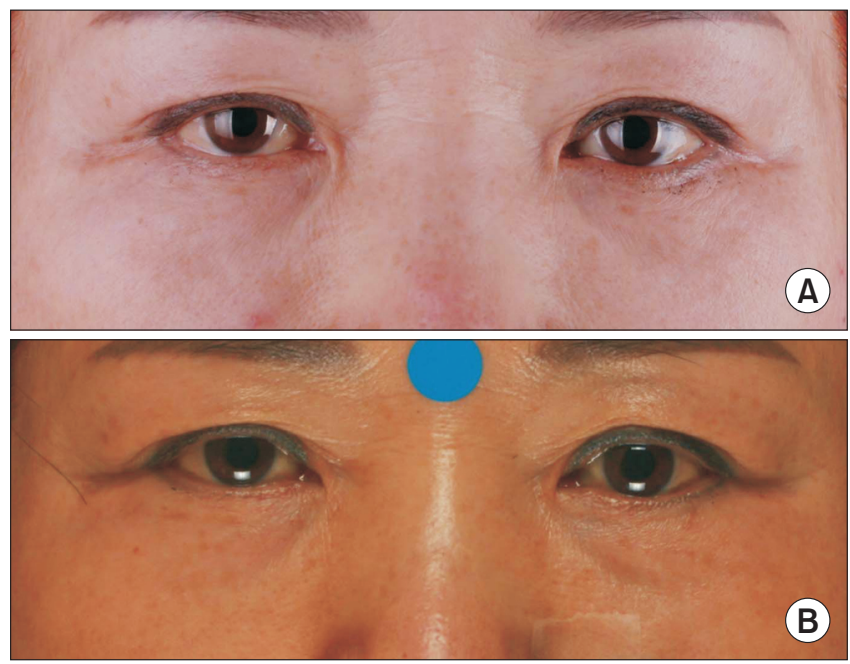

Fig. 3. Photos of case 2. (A) Before and (B) 2 weeks after the third sessions of Inus ${ }^{\mathrm{TM}}$ (8 weeks after the first session). were also greatly improved. These effects achieved in this clinical case were beyond expectations.

The maximized effect from the FMR is suspected to be due to the synergy obtained by the rejuvenated lower lid itself and the additional tightening of the soft tissues in the periorbital regions. On the other hand, the boundary between the lid cheek junctions became more distinct with the appearance of deep tear troughs. This phenomenon may be attributed to two of the following reasons: first, the skin of the tear trough was excessively thin, resulting in a reduced degree of improvement compared to the skin of the proximal midface and the lower lid; second, the lifting of the orbital and periorbital regions influenced the depth and texture of the tear trough (Fig. 3B).

A 59-year-old female who underwent TBL was treated with Inus $^{\mathrm{TM}}$ for the correction of scars and wrinkles in the lower eyelids (Fig. 4). A bulging of scar in the right lateral canthal region and the pretarsal atrophy with linear and broad scars were observed. The skin of the lower lid was stretched downward. Additionally, lid laxity with a round contour of the lid and blunted lateral canthal angles could be observed (Fig. 4A).

Post-treatment, the entire region of the lid had a more volumized and lifted appearance owing to the distinct improvement in the contour of the eyelids. The contour and position of the lower lid and lateral canthus were dramatically changed, as if a revision TLB with suborbicualris oculi fat lifting and lateral canthopexy had been performed [5]. In general, lifting does not occur solely as a result of skin thickening. Therefore, these results suggest a possible contribution from the complementary stimulation of the underlying muscles and fascia in the orbital

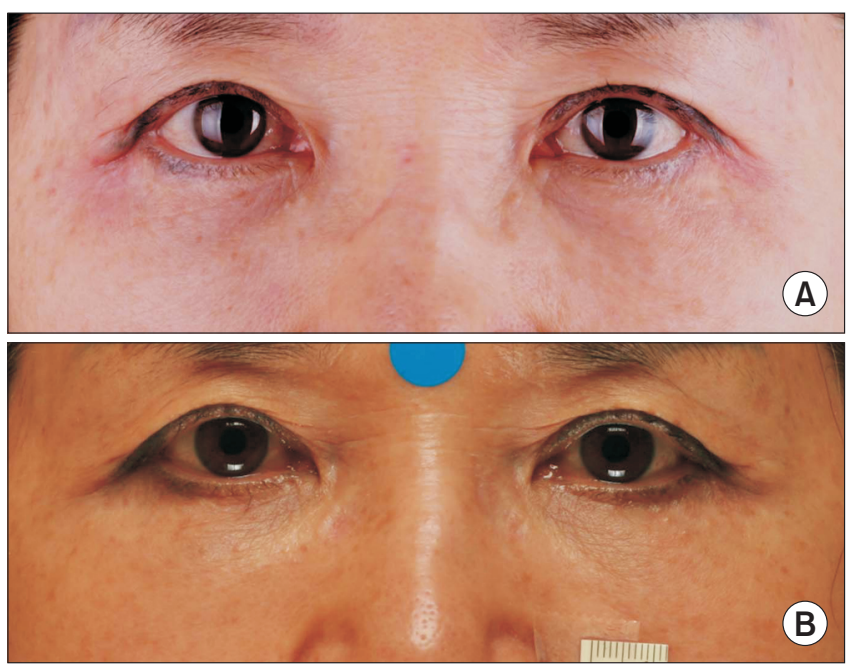

Fig. 4. Photos of case 3. (A) Before and (B) 2 weeks after the third sessions of Inus ${ }^{\mathrm{TM}}$ (8 weeks after the first session). 
and periorbital regions by the microneedle at a 1.0-mm depth.

The appearance of scars and wrinkles was also significantly reduced. The previously tense lateral canthal skin that stretched downwards was resolved. Moreover, great volume restorations around the lid-cheek junction and the proximal part of the midface, including the malar regions, similar to the effects of fat transplant or filler injections, were achieved. However, the pretarsal atrophy still persisted. This may be due to the excessive amount of pretarsal muscle removed during the previous TLB or the uneven distribution of volume restoration as a result of the inappropriate use of the Inus ${ }^{\mathrm{TM}}$ tip (Fig. 4B).

\section{Discussion}

In conclusion, skin rejuvenation by FMR was achieved by a combination of physical stimulation using microneedles and energy heating using RF current to promote wound healing [6]. To date, there have been several reports on FMR that claimed its potency in dermal remodeling, neoelastogenesis, and neocollagenogenesis, resulting in the collective efforts for dermal thickening [7]. Nevertheless, no study has been performed on the rejuvenation effect of the bipolar FMR on scars and wrinkles on the lower lid. No adverse events were reported in these three clinical cases except for temporary mild pain, erythema, and edema following the procedure.

In this report, the authors observed various benefits upon the application of FMR including the thickening of the skin and the improvement of wrinkles, scars, and ectropion as well as the lifting of the lower lid and modified eyelid contour. Despite the limitations with regard to the number of cases and the followup period, the findings suggest that FMR is a safe and effective skin rejuvenation method. A large-scale and a well-designed trial may be necessary to better investigate the efficacy and safety of FMR and to better provide guidelines for the treatment of the ocular and periocular regions.

\section{Supplementary materials}

Supplementary materials can be found via https://doi. org/10.25056/JCM.2021.5.2.99.

\section{Conflicts of interest}

The authors have nothing to disclose.

\section{References}

1. Kang KJ, Yang HH, Chai CY. Assessment of rejuvenation by change of lid-cheek junction after transconjunctival and traditional subciliary incision blepharoplasty. J Cosmet Med 2017;1:30-8.

2. Hruza G, Taub AF, Collier SL, Mulholland SR. Skin rejuvenation and wrinkle reduction using a fractional radiofrequency system. J Drugs Dermatol 2009;8:259-65.

3. Kim JK, Roh MR, Park GH, Kim YJ, Jeon IK, Chang SE. Fractionated microneedle radiofrequency for the treatment of periorbital wrinkles. J Dermatol 2013;40:172-6.

4. Shin JW, Park JT, Chae JB, Choi JY, Na JI, Park KC, et al. The efficacy of micro-insulated needle radiofrequency system for the treatment of lower eyelid fat bulging. J Dtsch Dermatol Ges 2019;17:149-56.

5. Kang KJ, Chai CG. Complementary effect of fat grafting on traditional subciliary incisional lower blepharoplasty: estimated by the change of lid-cheek junction. J Cosmet Med 2018;2:12-21.

6. Hwang UK, Lee JY, Kang KJ. Quantitative analysis of polydioxanone thread effects on lid-cheek junction. J Cosmet Med 2018;2:22-6.

7. Cho SI, Chung BY, Choi MG, Baek JH, Cho HJ, Park CW, et al. Evaluation of the clinical efficacy of fractional radiofrequency microneedle treatment in acne scars and large facial pores. Dermatol Surg 2012;38(7 Pt 1):1017-24. 